\section{Acute jaundice}

Andrew Burroughs FRCP

Lucy Dagher MD

Liver Transplantation \& Hepatobiliary

Medicine, Royal Free Hospital, London

Clin Med JRCPL 2001;1:285-9

The normal serum bilirubin concentration in adults is less than $18 \mu \mathrm{mol} / \mathrm{l}$. Jaundice is best looked for in the sclerae and mucous membrane of the soft palate and is a common sign in almost all liver and biliary tract diseases. There are several important questions of value in determining the diagnosis which the clinician should ask himself/herself:

1 Is the jaundice due to an obstruction of the biliary tree?

2 Is there evidence of chronic liver disease?

3 Is the jaundice possibly drug induced, alcohol induced or infective in origin?

4 Is there any evidence of haemolysis?

\section{Classification of jaundice (Fig 1)}

Jaundice can result from either an increase in bilirubin formation or a decrease in hepatic clearance. From a practical standpoint, it is reasonable to classify conditions that produce jaundice under three broader categories:

1 Isolated disorders of bilirubin metabolism.

2 Liver disease.

3 Obstruction of the bile ducts.

\section{Isolated disorders of bilirubin metabolism (Table 1)}

The mechanisms that can lead to isolated unconjugated hyperbilirubinaemia are:

- increased bilirubin production

- decreased hepatocellular uptake

- decreased bilirubin conjugation.

Hyperbilirubinaemia is often associated with a predominant elevation in indirect serum bilirubin concentration.
Liver function and the results of other standard liver tests are otherwise normal in all three conditions.

\section{Liver disease}

Generalised impairment of hepatocellular function may result from acute liver injury or chronic disease. A variety of disorders can produce acute or subacute hepatocellular injury including:

- viral hepatitis

- exposure to hepatotoxins

- hepatic ischaemia

- certain metabolic derangements.

Jaundice appears over days or weeks. Fatigue and malaise are common. There may be varying degrees of decompensation due to cirrhosis or just cutaneous markers of chronic liver disease. Signs of hepatic encephalopathy, if present, may be personality change, and in more severe cases manifest as flapping tremor, confusion and coma. Fluid retention in its mildest form may be exhibited as weight gain, but in severe forms oedema and ascites may be present. Bruising may be spontaneous or at sites of venepuncture. Serum biochemistry shows increase in transaminases, while serum albumin levels may be low and prothrombin time prolonged.

\section{Obstruction of the bile ducts}

The bile ducts may be obstructed because of mechanical obstruction of the biliary tree by the presence of stones or by intrinsic disorders of the bile duct and extrinsic compression, most commonly cholangiocarcinoma and carcinoma of the pancreas, respectively. Without mechanical obstruction the most common causes of cholestatic jaundice are drug induced and following hepatitis A. The patient does not have signs of chronic liver disease but may have signs indicating malignancy. Pruritus is usual, and the patient becomes increasingly pigmented. There are raised levels of conjugated bilirubin, biliary alkaline phosphatase and total cholesterol in the

Fig 1. Classification of jaundice.

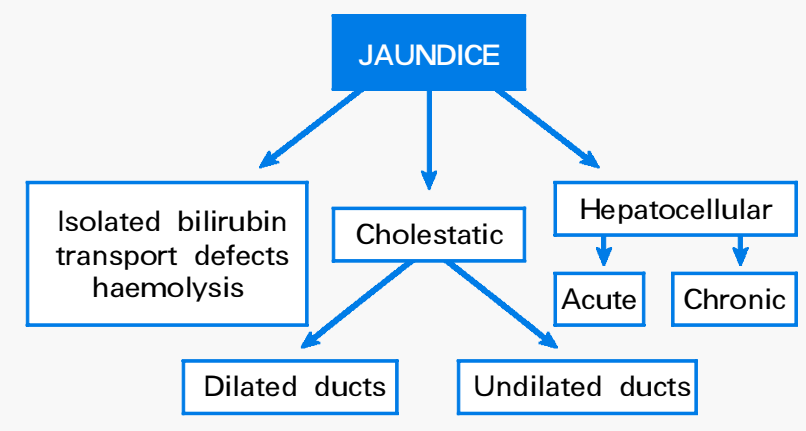

Table 1. Disorders of bilirubin metabolism.

Unconjugated hyperbilirubinaemia:

Increased bilirubin production

Decreased hepatocellular uptake

Decreased conjugation

Conjugated or mixed hyperbilirubinaemia
Haemolysis

Ineffective erythropoiesis

Blood transfusion

Resorption of haematomas

Drugs (eg rifampicin)

Gilbert syndrome

Gilbert syndrome

Crigler-Najar syndrome

Physiologic jaundice of the newborn

Dubin-Johnson syndrome

Rotor syndrome 


\section{Key Points}

Scleral jaundice can be detected if serum bilirubin is above $34 \mu \mathrm{mol} / \mathrm{l}$, but more commonly $50 \mu \mathrm{mol} / \mathrm{l}$ is the threshold

A careful history, physical examination and review of the standard laboratory tests should allow a physician to make an accurate diagnosis in $85 \%$ of cases

In acute jaundice, liver ultrasound should be the first imaging modality

If there is evidence of biliary obstruction and a therapeutic intervention is planned, endoscopic retrograde cholangiopancreatography or percutaneous transhepatic cholangiography is the investigation of choice

Magnetic resonance cholangiopancreatography should follow ultrasound if the latter has been technically difficult, if obstruction has not been ruled out or therapeutic intervention is not needed

Liver biopsy is needed to confirm the presence, cause and severity of chronic liver disease

serum. Steatorrhoea is responsible for weight loss and malabsorption of calcium and fat-soluble vitamins A, D, E and $\mathrm{K}$.

In adult patients with new onset of jaundice, eight disorders account for $98 \%$ of the diagnoses ultimately established $^{1}$ :

- viral hepatitis

- alcoholic liver disease

- chronic hepatitis

- drug induced liver diseases

- gallstones and their complications

- carcinoma of the pancreas

- primary biliary cirrhosis (PBC)

- primary sclerosing cholangitis.

By the time patients with metastatic liver disease develop jaundice, the diagnosis is usually obvious because the liver has been extensively replaced by tumour tissue.

Careful history, physical examination and review of standard laboratory tests should allow a physician to make an accurate diagnosis in $85 \%$ of cases ${ }^{1}$.

\section{History (Fig 2)}

An accurate clinical history is particularly important in differentiating the various causes of jaundice. In conjunction with examination, it determines the most appropriate investigations.

The increase in foreign travel and migration to developed countries have increased exposure not only to viral hepatitis but also to a greater number of rarer causes of jaundice such as malaria, and inherited haemoglobinopathies. A family history is important with regard to heritable disorders of bilirubin metabolism such as Gilbert syndrome, chronic liver disease such as Wilson's disease, haemochromatosis and autoimmune conditions which may be associated with chronic active hepatitis or PBC. Special attention should be paid to identifying groups at particular risk of contracting infective viral hepatitis such as intravenous (IV) drug abusers, homosexuals, following needle-stick injuries or contact with jaundiced individuals, and patients who have received blood transfusions.

Drugs and environmental hepatotoxins may not only be hepatotoxic but

Fig 2. History taking in acute jaundice.

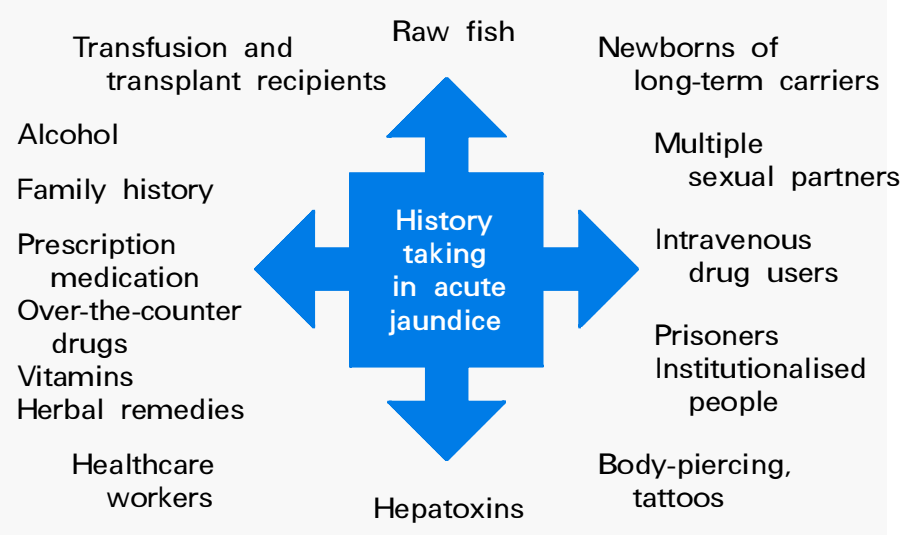

some can cause haemolysis. Drug ingestion may need to be sought by searching the patient's home and workplace and by re-taking the history as well as checking general practitioner (GP) records. Ingestion of significant quantities of paracetamol or the mushroom Amanita phalloides may lead to hepatocellular necrosis and jaundice within several days of exposure ${ }^{2}$. Toxic liver injury can have a fulminant course associated with a high mortality. Several drugs can produce idiosyncratic hepatocellular injury and jaundice, the most common being nonsteroidal anti-inflammatory drugs (NSAIDs), amiodarone, terbinafine, isoniazid, methyldopa, phenytoin and the inhalational anaesthetic halothane. Excessive alcohol intake is especially important because it is common; a detailed history of alcohol consumption should be taken from the patient, relatives and the GP. Alcoholic hepatitis should be a diagnostic consideration in the jaundiced patient with ethanol dependency, particularly when hepatomegaly and fever are present.

\section{Physical examination}

\section{Signs and symptoms}

A history of fever, especially accompanied by shaking chills, or abdominal pain particularly in the right upper quadrant, is suggestive of cholangitis caused by obstructive diseases (particularly choledocholithiasis), as is a history of biliary surgery. Symptoms compatible with viral prodrome, such as anorexia, 
Table 2. Signs and symptoms of viral hepatitis.

\section{Infection}

Short-term (acute)

Tiredness, anorexia, malaise, myalgias

Nausea or stomach ache

Diarrhoea

Skin rash

Yellow eyes/skin (jaundice)

Light-coloured stools

Dark yellow urine

malaise and myalgias point to viral hepatitis (Table 2). Systemic features of malignancy should be sought (eg weight loss, anaemia, anorexia), especially if of short duration. These symptoms in conjunction with painless jaundice, particularly in a middle-aged or older person, suggest neoplasm of the head of the pancreas.

Scleral jaundice can be detected if serum bilirubin is above $34 \mu \mathrm{mol} / \mathrm{l}$, but more commonly $50 \mu \mathrm{mol} / \mathrm{l}$ is the threshold. Needle marks should suggest IV drug abuse. Skin excoriations confirm that the patient has been scratching; this may be particularly severe in patients with PBC and primary sclerosing cholangitis. Three physical findings indicate that a patient is almost certainly drinking excessive amounts of alcohol:

- parotid enlargement

- gynaecomastia

- Dupuytren's contracture.

Evidence of portal hypertension (ie ascites, splenomegaly, prominent abdominal veins) and/or spider angiomata, gynaecomastia and asterixis suggest the presence of chronic parenchymal liver disease. Certain physical findings may suggest particular liver diseases, for example pigmentation in chronic biliary disease and haemochromatosis, xanthomas in PBC, and Kaiser-Fleischer rings in Wilson's disease.

Dilated peri-umbilical veins indicate a portal collateral circulation in cirrhosis, while ascites may be due to cirrhosis or malignant disease. A very large nodular liver suggests cancer. A small liver may indicate severe hepatitis or cirrhosis, excluding intrahepatic cholestasis in

\section{Long-term \\ (chronic)}

\begin{abstract}
Same symptoms as acute Muscle and joint ache Weakness

Signs \& symptoms of cirrhosis

Signs \& symptoms of liver cancer

Secondary amenorrhoea
\end{abstract}

which the liver is usually enlarged. The edge is tender in hepatitis, congestive liver failure, bacterial cholangitis, occasionally in malignant liver disease, and with alcoholic hepatitis or severe fatty liver. An arterial murmur over the liver indicates acute alcoholic hepatitis, liver cancer or an arteriovenous fistula.

\section{Investigations (Fig 3)}

The pattern of the liver enzyme tests may be helpful, with additional testing to identify a cause of jaundice. The ratio of aspartate aminotransferase to alanine aminotransferase appears to be a useful index for distinguishing non-alcoholic steatohepatitis (NASH) from alcoholic liver disease. Although ratios less than 1 suggest NASH or viral hepatitis, a ratio of 2 or above strongly suggests alcoholic liver disease $\mathrm{e}^{3,4}$. Gamma-glutamyl transferase elevation strengthens the diagnosis of alcohol abuse. A drug effect is possible if there is a correlation between the onset of jaundice, elevation of liver enzymes and the start of drug administration. The drugs that most commonly cause transaminase abnormalities are NSAIDs, HMG-CoA reductase inhibitors, antiepileptic drugs and antituberculosis drugs. In addition to prescribed medication, over-the-counter medications, herbal preparations and illicit drug use may also be the cause. The first step to determine whether a medication or alcohol is responsible is to stop the potential injurious agent and see whether the tests return to normal.

If the abnormalities persist, particularly if transaminase values remain high, markers of autoimmune disease should

Fig 3. An algorithm for investigation in the patient with acute jaundice (AMA = antimitochondrial antibody; $C T=$ computed tomography; $E R C P=$ endoscopic retrograde cholangiopancreatography; $\mathrm{MRCP}=$ magnetic resonance cholangiopancreatography; $M R I=$ magnetic resonance imaging).

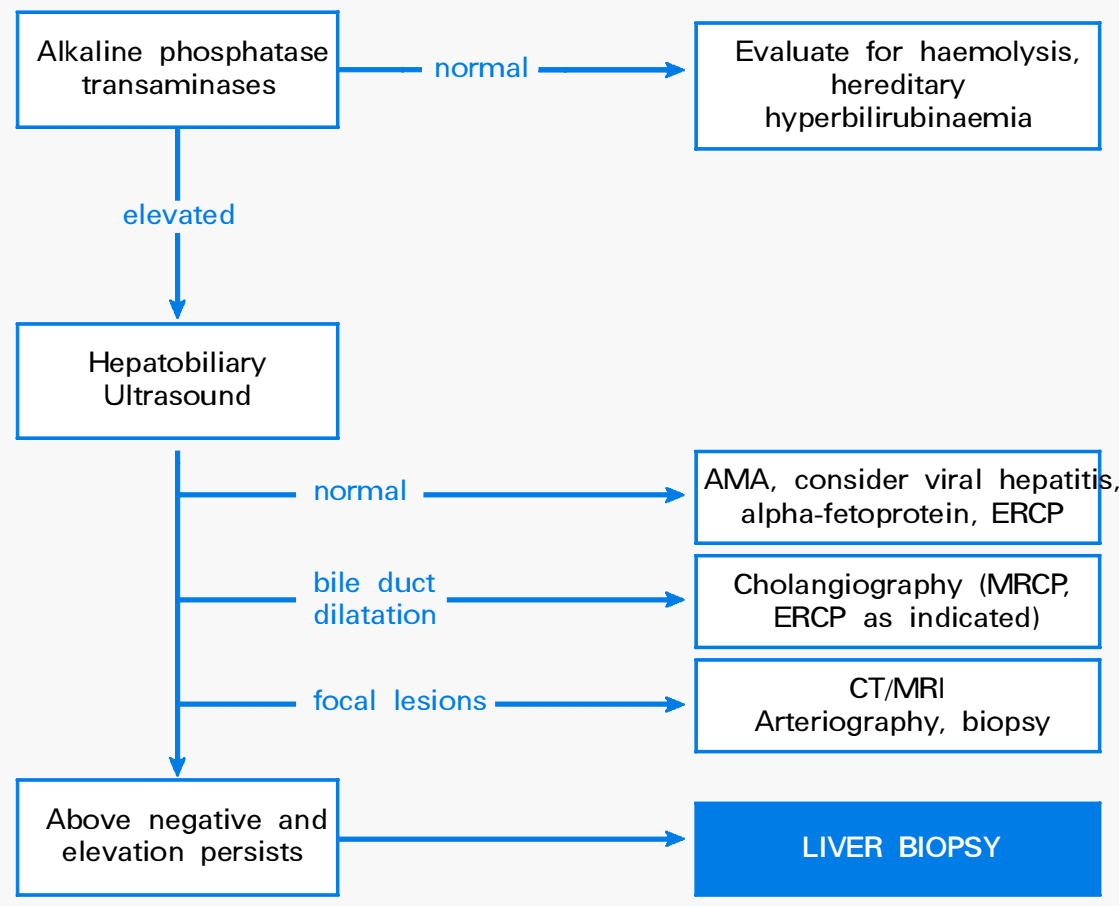


be sought, especially in young women. Serologic studies for hepatitis B and $\mathrm{C}$ should be done in all patients, and iron studies routinely obtained to assess the possibility of haemochromatosis. Caeruloplasmin and copper levels are appropriate routine tests in patients up to the age of 45 years to detect Wilson's disease. Alpha 1-antitrypsin levels should be evaluated if the above measurements fail to explain the aetiology of persistently raised aminotransferases.

All patients with persistent jaundice of uncertain cause should be advised to discontinue unnecessary medications and abstain from alcohol for several weeks and then be tested again. If the alkaline phosphatase is elevated, ultrasound scanning should be performed to exclude biliary obstruction and space-occupying lesions. A normal ultrasound suggests chronic cholestatic or infiltrative disease.

\section{Radiology}

\section{Liver ultrasound}

The sensitivity of ultrasound to dilatation of the bile ducts has made it the imaging technique of choice in the evaluation of jaundice ${ }^{5}$. If clinical history and laboratory tests suggest obstructive aetiology in acute jaundice, liver ultrasound should be the first imaging modality and it is considered the 'stethoscope' of the hepatologist. It can confirm or exclude obstruction, signs of chronic liver disease and space-occupying lesions. The sensitivity of abdominal ultrasonography for the detection of biliary obstruction in jaundiced patients ranges from $55-91 \%$ and the specificity from $82-95 \%^{2}$. Ultrasonography can also demonstrate cholelithiasis and space-occupying lesions greater than $1 \mathrm{~cm}$ in diameter. The major advantages of ultrasound are that it is non-invasive, portable and relatively inexpensive. The major disadvantages are that it is operator dependent and the images may be difficult to interpret in obese patients or in those with overlying bowel gas. Also, dilatation of the common bile duct, which usually indicates biliary tract obstruction, is often present in patients who have undergone previous cholecystectomy.

\section{Computed tomography of the abdomen}

Abdominal computed tomography (CT) permits accurate measurements of the calibre of the biliary tree, with a sensitivity of $63-96 \%$ and a specificity of 93-100\% for detecting biliary obstruction $^{2}$. Spiral (helical) CT improves the diagnostic accuracy of this method. Abdominal CT also detects spaceoccupying lesions as small as $5 \mathrm{~mm}$. Unlike ultrasonography, CT is not operator dependent and it provides technically superior images in obese patients in whom the biliary tree is obscured by bowel gas. CT is not as accurate as ultrasonography in detecting cholelithiasis because only calcified stones can be seen clearly. Other considerations in the use of abdominal CT in patients with jaundice are its lack of portability, requirement of IV contrast and expense.

\section{Endoscopic retrograde cholangiopancreatography}

Direct visualisation of the biliary tree as well as the pancreatic ducts is possible with endoscopic retrograde cholangiopancreatography (ERCP). ERCP is more invasive than abdominal ultrasonography and CT and requires conscious sedation. It is highly accurate in the diagnosis of biliary obstruction, with a sensitivity of $89-98 \%$ and a specificity of $89-100 \%{ }^{6}$. In addition to providing radiographic images, biopsy specimens and brushings for cytology or periampullary lesions can be obtained at ERCP. Moreover, if a focal cause of biliary obstruction is identified (eg choledocholithiasis, stricture), manoeuvres to relieve the obstruction (eg sphincterectomy, stone extraction, dilatation, stent placement) can be performed during the same session. The rates of morbidity and mortality with ERCP from untoward events such as respiratory depression, aspiration, bleeding, perforation, cholangitis and pancreatitis are $3 \%$ and $0.2 \%$, respectively $^{7}$. A final consideration is cost: ERCP is more expensive than non-invasive procedures.

\section{Percutaneous transhepatic cholangiography}

Percutaneous transhepatic cholangiography (PTC) complements ERCP. Its sensitivity (98-100\%) and specificity (89-100\%) for the diagnosis of biliary tract obstruction are comparable with those of ERCP ${ }^{7}$. Like ERCP, it is recommended when interventional procedures are needed, such as balloon dilatation and stent placement to relieve amenable focal obstructions of the biliary tree. PTC is preferred when the level of biliary obstruction is proximal to the common hepatic duct or where altered anatomy precludes ERCP. PTC may be technically limited in the absence of dilatation of the intrahepatic bile ducts. Morbidity (3\%) and mortality $(0.2 \%)$ of PTC are due to bleeding, perforation and cholangitis.

\section{Magnetic resonance imaging}

Magnetic resonance imaging is an increasingly useful investigation of hepatobiliary disease, especially in diagnosing and staging malignant disease. Alterations in weighting of the image lead to prominence of the ductal system in the biliary tree and pancreas, known as magnetic resonance cholangio pancreatography (MRCP). The basic principle of MRCP is to utilise T2weighted images in which stationary or slowly moving fluid, including bile, is high in signal intensity and all surrounding tissues, including retroperitoneal fat and solid visceral organs, are lower in signal. MRCP is a promising new tool that provides detail of the liver parenchyma and biliary tree ${ }^{8}$. MRCP can be employed:

- when there are contraindications to or failure of ERCP or PTC

- if therapeutic intervention is unlikely to be required

- as a first imaging test when there is a previous biliary-enteric or Billroth II anastomosis $^{9,10}$.

MRCP has a 95\% sensitivity for detecting obstruction, though it is 
inaccurate in assessing the grade of obstruction $^{11,12}$. Similarly, strictures cannot be well characterised due to a signal drop out. Its accuracy is equal to that of ERCP in determining both the level of obstruction and whether the obstruction is due to a neoplastic process $^{10}$. Unlike ERCP or PTC, MRCP enables the biliary tree to be visualised above and below a complete obstruction. It has an advantage over ERCP in being non-interventional, non-operator dependent, and does not require contrast injection although, unlike ERCP, it is purely diagnostic. MRCP has already replaced direct cholangiography in many clinical circumstances, but ERCP and PTC remain the tests of choice when a therapeutic intervention is necessary.

\section{Further studies}

\section{Serologic testing}

When imaging studies do not suggest biliary obstruction, evaluation for underlying liver disease must be undertaken in jaundiced patients with biochemical evidence of hepatocellular dysfunction or cholestasis. Appropriate screening includes:

- viral serologic testing (for hepatitis A, B and C)

- serum levels of iron, transferrin and ferritin (for haemochromatosis), caeruloplasmin and copper (for Wilson's disease)

- antimitochondrial antibodies (for PBC)

- antinuclear antibodies

- smooth muscle antibody (for autoimmune hepatitis)

- alpha 1-antitrypsin (for alpha 1antitrypsin deficiency).
Confirmation of these diagnoses, as well as elucidation of diagnoses not revealed by serologic analysis, may be made by liver biopsy.

\section{Liver biopsy}

Liver biopsy provides information about hepatic inflammation and lobular architecture. It is most helpful in undiagnosed persistent jaundice and to stage the chronic liver disease. With special histologic features, special stains and/or quantification of copper or iron content, it permits the diagnosis of a number of liver diseases, including:

- viral and alcoholic hepatitis

- Wilson's disease

- haemochromatosis

- alpha 1-antitrypsin deficiency

- fatty liver of pregnancy

- PBC

- granulomatous hepatitis

- neoplasms.

Liver biopsy carries a small complication rate, predominantly from bleeding and perforation, with morbidity less than $0.5 \%$ and a mortality of $0.017 \%{ }^{13}$.

\section{References}

1 Greenberger N. History taking and physical examination in patients with liver disease. In: Schiff E, Sorrell M, Maddrey WC (eds). Schiff's diseases of the liver. Philadelphia: Lippincot-Raven Publishers, 1999:193-204.

2 Lidofsky S, Scharschmidt BF. Jaundice. In: Feldman M, Schsrschmidt BF, Sleisenger $\mathrm{MH}$ (eds). Sleisenger and Fordtran's Gastrointestinal and liver disease. Philadelphia: Saunders, 1998:220-32.

3 Cohen JA, Kaplan MM. The SGOT/SGPT ratio: an indicator of alcoholic liver disease. Dig Dis Sci 1979;24:835-8.
4 Sorbi D, Boynton J, Lindor KD. The ratio of aspartate aminotransferase to alanine aminotransferase: potential value in differentiating nonalcoholic steatohepatitis from alcoholic liver disease. Am J Gastroenterol 1999;94:1018-22.

5 Barloon TJ, Bergus GR, Weissman AM. Diagnostic imaging to identify the cause of jaundice. Am Fam Physician 1996;54: 556-62.

6 Parsanen PA, Partanen KP, Pikkarainen PH, Alhava FM et al. A comparison of ultrasound, computed tomography and endoscopic retrograde cholangiopancreatography in the differential diagnosis of benign and malignant jaundice and cholestasis. Eur J Surg 1993;159:20-9.

7 Cotton PB. Critical appraisal of therapeutic endoscopy in biliary tract diseases. Review. Annu Rev Med 1990;41:211-22.

8 Taourel P, Bret PM, Reinhold C, Barkun AN, Atri M. Anatomic variants of the biliary tree: diagnosis with MR cholangiopancreatography. Radiology 1996;199:521-7.

9 Barish MA, Soto JA. MR cholangiopancreatography: techniques and clinical applications. Review. Am J Roentgenol 1997; 169:1295-303.

10 Soto JA, Barish MA, Yucel EK, Siegenberg $\mathrm{D}$, et al. Magnetic resonance cholangiography: comparison with endoscopic retrograde cholangiopancreatography. Gastroenterology 1996;110:589-97.

11 Guibaud L, Bret PM, Reinhold C, Atri M, Barkun AN. Bile duct obstruction and choledocholithiasis: diagnosis with MR cholangiography. Radiology 1995;197: 109-15.

12 Morimoto K, Shimoi M, Shirakawa T, Aoki $\mathrm{Y}$, et al. Biliary obstruction: evaluation with three-dimensional MR cholangiography. Radiology 1992;183:578-80.

13 Grant A, Neuberger J. Guidelines on the use of liver biopsy in clinical practice. British Society of Gastroenterology. Gut 1999; 45(Suppl 4):IV1-11.

Address for correspondence:

Dr Andrew Burroughs, Consultant

Physician and Hepatologist, Royal

Free Hospital, Pond Street, London NW3 2QG 